\title{
Investigation of the Effectiveness of Intelligence-Firepower Method of Defeat Radiating Radio-Electronic Facilities
}

Maksim L. Parinov, Sergei V. Petrenkov and Ervand A. Mamajanyan* Military Training and Research Center of the Air Force «Air Force Academy ft. Professor N.E. Zhukovsky and Y.A. Gagarin» 54a Starykh Bol'shevikov Str., Voronezh, 394064, Russia

Received 01.08.2015, received in revised form 21.09.2015, accepted 09.03.2016

The method allowing to evaluate the efficiency of the process of destruction of the radiating radioelectronic facility according to radio intelligence tools with consistent review. The results of the study allow to evaluate the effectiveness of each component of the modeled process and formulate requirements to the subsystems for given values of radio-electronic destruction of the object.

Keywords: the probability of hitting, radar intelligence with consistent review.

\section{Исследование эффективности}

разведывательно-огневого метода поражения

излучающих радиоэлектронных объектов

\author{
М.Л. Паринов, С.В. Петренков, Е.А. Мамаджанян \\ ВУНЦ ВВС «ВВА им. проф. Н.Е. Жуковского и Ю.А. Гагарина» \\ Россия, 371600, Воронеж, ул. Старых Большевиков, $54 f$
}

Предложен метод, позволяющий оченить эффективность процесса поражения излучающего радиоэлектронного объекта по данным радиоразведки с последовательным обзором. Результаты исследования позволяют как оценить эффективность каждой составляющей моделируемого процесса, так и сформировать требования к подсистемам по заданным значениям показателя уничтожения радиоэлектронного объекта.

Ключевые слова: вероятность поражения, радиоразведка с последовательным обзором.

(C) Siberian Federal University. All rights reserved

* Corresponding author E-mail address: sema1709@yandex.ru 
Анализ локальных войн и вооруженных конфликтов показывает значительное увеличение составляющей высокоточных средств поражения, предназначенных в том числе для уничтожения (вывода из строя) радиоэлектронных объектов противника. Данные обстоятельства, в свою очередь, накладывают жесткие требования к информационной компоненте обеспечения высокоточного поражения как по оперативности, так и по точности вскрытия пространственного размещения уничтожаемых объектов.

Изложенное свидетельствует о высокой интеграции и тесной взаимосвязи информационной и огневой составляющих вооруженного конфликта, что предопределяет возможные взаимные ограничения на количественные значения показателей эффективности каждой из рассматриваемых компонент.

Исходя из этого, целью настоящей статьи является разработка метода, позволяющего с системных позиций реализовать учет возможного влияния разведывательной составляющей информационного обеспечения на процесс огневого поражения радиоэлектронных объектов противника.

Наиболее целесообразно в качестве показателя эффективности огневого поражения излучающего радиоэлектронного средства РЭС определить вероятность поражения $P_{\text {пор }}(t)$ как величину, отражающую реализацию основного функционала средств поражения и фактически являющуюся оценкой возможности по уничтожению радиоэлектронного объекта РЭОб.

Структурная модель огневого воздействия на РЭОб, сформированная исходя из особенностей внутрисистемных взаимосвязей элементов рассматриваемого процесса, представлена на рис. 1.

Исходя из структуры представленной модели поражения, динамическую модель сформируем в виде ориентированного графа состояний (рис. 2).

На рис. 2 приняты следующие обозначения:

$C_{0}$ - исходное состояние;

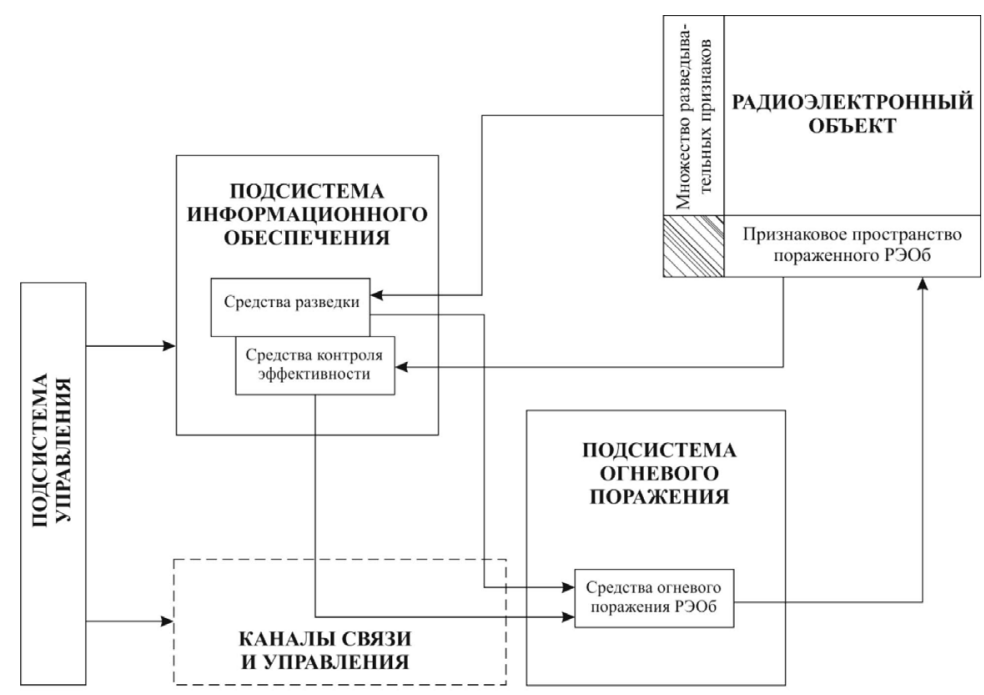

Рис. 1. Структурная модель процесса поражения излучающих РЭС 


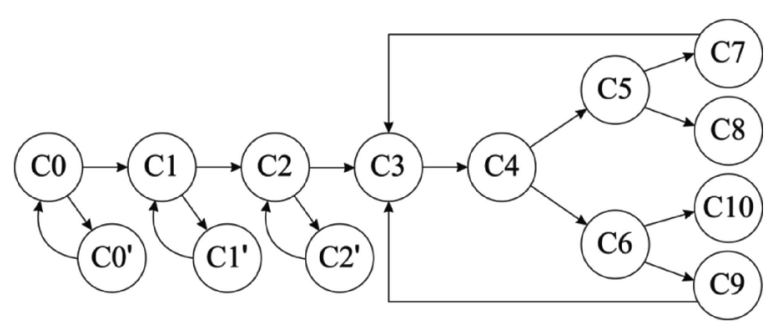

Рис. 2. Ориентированный граф состояний, отражающий динамику комплексного поражения РЭС

$C_{1}$ - поиск и обнаружение РЭС (РЭС обнаружено);

$C_{0}{ }^{\prime}-$ РЭС не обнаружено;

$C_{2}$ - высокоточное определение координат РЭС, подлежащих поражению (оценка координат РЭС произведена);

$C_{l}{ }^{\prime}-$ оценка координат РЭС не произведена;

$C_{3}$ - передача информации на огневые средства (артиллерийские батареи) поражения (информация на средства поражения передана);

$C_{2}{ }^{\prime}-$ информация на средства поражения не передана;

$\mathrm{C}_{4}$ - попадание снаряда в заданный сектор;

$C_{5}-$ РЭС не поражено;

$C_{6}$ - РЭС поражено;

$C_{7}$ - принятие решения о том, что цель не поражена при ее непоражении;

$C_{8}$ - принятие решения о том, что цель поражена при ее непоражении;

$C_{9}$ - принятие решения о том, что цель не поражена при ее поражении;

$C_{10}$ - принятие решения о том, что цель поражена при ее поражении.

В качестве математической модели процесса поражения рассмотрим полумарковский процесс, протекающий на множестве состояний представленного ориентированного графа.

Введем в рассмотрение передаточную функцию перехода графа $\left(C_{i} \rightarrow C_{j}\right)$ в следующем виде:

$$
h_{i j}(s)=L_{s}\left\{P_{i j} \varphi_{i j}(\mathrm{t})\right\},
$$

где $P_{i j} \varphi_{i j}(\mathrm{t})$ - вероятность и плотность вероятности распределения времени ожидания при переходе из $i$-е в $j$-е состояние соответственно [1].

В соответствии с передаточной функцией перехода сформируем динамическую модель процесса поражения в виде передаточных функций (рис. 3) [1, 2].

Исходя из топологической структуры модели интервальные вероятности для состояний, характеризующего поражение цели, получим следующее аналитическое соотношение:

$$
\begin{gathered}
\widetilde{P}_{10}(s)=\frac{P_{0 h} h_{0,1}(s) h_{1,2}(s) h_{2,3}(s) h_{3,4}(s) h_{4,6}(s) h_{6,10}(s)}{\left(1-h_{0,0^{\prime}}(s) h_{0^{\prime}, 0}(s)\right)\left(1-h_{1,1}(s) h_{1^{\prime}, 1}(s)\right)\left(1-h_{2,2^{\prime}}(s) h_{2,2}(s)\right)} \times \\
\times \frac{1}{1-\left(h_{3,4}(s) \cdot\left(h_{4,5}(s) h_{5,7}(s) h_{7,3}(s)+h_{4,6}(s) h_{6,9}(s) h_{9,3}(s)\right)\right)} . \\
-343-
\end{gathered}
$$




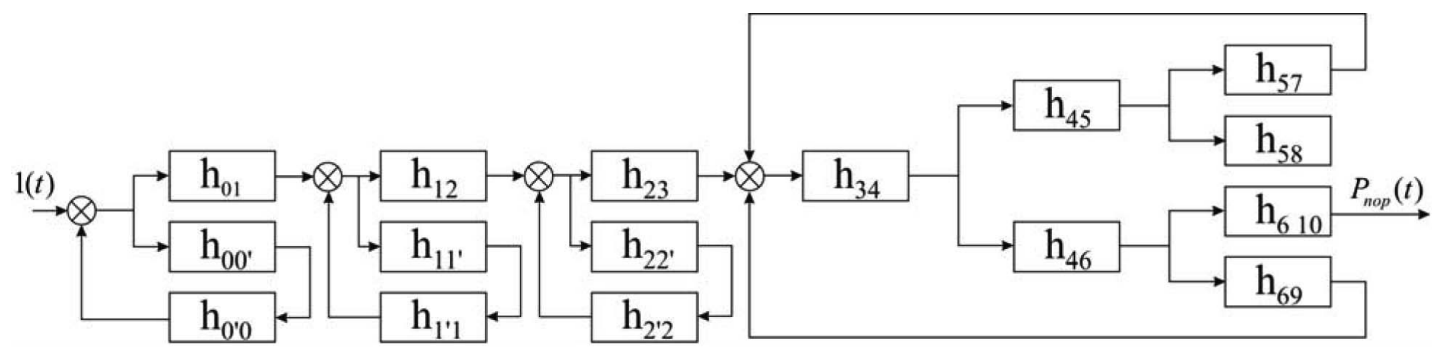

Рис. 3. Динамическая модель процесса поражения

Окончательно искомая вероятность при единичном ступенчатом воздействии может быть представлены в виде

$$
(t)=L_{s}^{-1}\left[\frac{1}{s} \widetilde{P}_{10}(s)\right] .
$$

В интересах обоснования множества исходных данных рассмотрим более детально каждое из состояний приведенного выше графа.

Переход $C_{0} \rightarrow C_{1}$ связан с вероятностно-временными характеристиками поиска РЭС и выделения истинной цели среди совокупности ложных целей в условиях непреднамеренных и преднамеренных помех.

Поисковую составляющую моделируемого процесса рассмотрим на основании реализации триангуляционно-корреляционного метода местоопределения [3, 4].

Способ заключается в реализации синхронного по пространству и времени пеленгования источников излучения с последующей корреляционной обработкой потока сигналов от каждого из пеленгаторов. Пространственно-временная синхронизация реализуется путем одновременного формирования диаграмм направленности пеленгаторов, направления максимумов которых ориентированы на геометрический центр просматриваемого элемента области поиска.

Для решения задачи поиска истинной цели допустим, что в секторе поиска находится одно РЭС, $P_{D}(t)$ - вероятность правильного обнаружения, $T_{a}$ - время анализа элемента разрешения, $M=M_{x} \times M_{y}$ - число элементов поиска, $P(x, y)$ или $P_{i}^{k}-$ вероятность попадания в элемент разрешения.

Опираясь на результаты, полученные в [2], безусловную вероятность правильного обнаружения сигнала определим в следующем виде:

$$
P_{D}=\sum_{k=1}^{M} D_{k} \cdot P_{i}^{k} \cdot \prod_{j=1}^{k-1} \hat{F}_{j} \cdot\left(1-P_{i}^{j}\right)
$$

Для случая равновероятного распределения с перераспределением вероятностей от элемента к элементу сектора поиска $P_{i}^{k}=\frac{1}{m-k+1}$, принимая во внимание неизменность условных вероятностей $D$ и $\hat{F}$ в каждом из элементов разрешения, получим: 


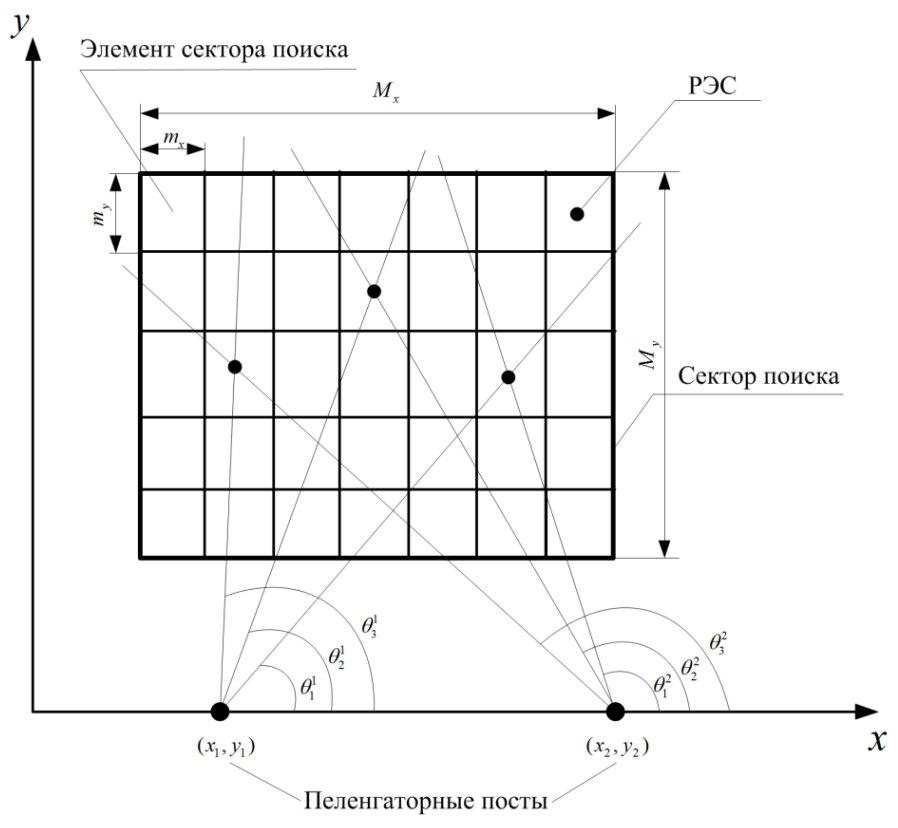

Рис. 4. Триангуляционно-корреляционный метод местоопределения

$$
P_{D}=\frac{D}{m} \sum_{k=1}^{M} \hat{F}^{k-1}
$$

С учетом времени, затрачиваемого на принятие решения при анализе, окончательное выражение для вероятности правильного обнаружения запишем в виде

$$
P_{D}(t)=\frac{D}{m} \sum_{k=1}^{M} \hat{F}^{k-1} \cdot h\left(t-k T_{a}\right),
$$

где $h(t)-$ функция Хэвисайда.

Для перехода $C_{0} \rightarrow C_{1}$ приближенно можно воспользоваться финальной вероятностью и средним временем обнаружения, при этом положив:

$$
\begin{aligned}
& \varphi_{01}(t)=\delta\left(t-\bar{T}_{\text {обн }}\right), \\
& P_{01}=P_{0 D}=\lim _{t \rightarrow \infty} P_{D}(t) .
\end{aligned}
$$

Путем несложных математических преобразований с учетом результатов, изложенных в [2], исходя из (6), для $\bar{T}_{\text {обн }}$ и $P_{0 D}$ находим:

$$
\begin{aligned}
& \bar{T}_{\text {обн }}=T_{a}\left[\frac{1-(M-1) \hat{F}^{M}+M \hat{F}^{M+1}}{(1-\hat{F})\left(1-\hat{F}^{M}\right)}\right], \\
& P_{01}=P_{0 D}=\frac{D}{m} \cdot \frac{\hat{F}^{M}-1}{\hat{F}-1} .
\end{aligned}
$$

Выражения (8) и (9) описывают процесс поиска и учитывают все составляющие этого процесса. 
Рассмотрим переход $C_{1} \rightarrow C_{2}$, который непосредственно связан с оценкой координат источника радиоизлучения. Время, затрачиваемое на оценку координат, представляет собой детерминированную величину в силу неизменности условия просмотра сектора поиска от цикла к циклу. Однако в этом случае возникает методическая сложность в определении вероятности перехода. Если в основу физической трактовки перехода положить событие, состоящее в том, что оценка местоположения излучающих РЭС будет осуществлена с точностью не хуже заданной по координатам $x$ и $y$, то вероятность этого события можно использовать в качестве переходной вероятности $C_{1} \rightarrow C_{2}$.

Определим указанную вероятность. С этой целью воспользуемся распределением ошибки определения местоположения излучающих РЭС по соответствующим координатам. При этом будем полагать, что оценка ошибки несмещенная, среднее значение ошибки приравниваем к нулю ( $\left.m_{x}=m_{y}=0\right)$, а истинное положение излучающего РЭС совпадает со средним значением ошибки. Кроме того, предположим, что воздействие помех сказывается лишь на дисперсии распределения ошибок по координатам $x$ и $y$.

В этом случае произведение переходной вероятности на плотность распределения времени перехода из состояния $C_{l}$ в состояние $C_{2}$ определяется выражением

$$
h_{12}(s)=P_{12} \varphi_{12}(s)=\left[1-2 F\left(\frac{h_{x}}{\sigma_{x}}\right)\right]\left[1-2 F\left(\frac{h_{y}}{\sigma_{y}}\right)\right] \cdot \exp \left(-s \bar{T}_{0}\right)
$$

где $\bar{T}_{0}$ - время, затрачиваемое на оценку координат излучающего РЭС после его обнаружения; $\sigma_{x}^{2}, \sigma_{y}^{2}$ - дисперсии ошибок местоопределения, которые рассчитываются исходя из особенностей триангуляционно-корреляционного метода координатометрии источника радиоизлучения $[5,6] ; F(x)$ - функция Лапласа плотности распределения ошибки местоопределения.

Таким образом, полученные выражения характеризуют с вероятностной точки зрения переход $C_{1} \rightarrow C_{2}$ и учитывают точностные и динамические возможности при оценке местоположения излучающего РЭС.

Переход $C_{2} \rightarrow C_{3}$ связан с подготовкой и передачей информации на средства огневого поражения. Время передачи информации будет зависеть от используемых для этой цели средств, а также от сложившейся помеховой обстановки.

Передача сообщений реализуется с помощью помехоустойчивого кодирования.

Усредненная вероятность битовой ошибки вычисляется по формуле

$$
P_{B}=\frac{1}{n} \sum_{i=t+1}^{n}(i+t) C_{n}^{i} p^{i}(1-p)^{n-i},
$$

где $C_{n}^{i}=\frac{n !}{i !(n-i) !} ; n-$ общее число бит информации; $t-$ число исправляемых битовых ошибок; $p$ - вероятность ошибочного приема канального символа, которая определяется типом сигнала. К примеру, для сигнала BFSK

$$
p=\frac{1}{2} \cdot \exp \left(-\frac{q}{2}\right),
$$
где $q=\frac{E_{c}}{N_{0}} \cdot \frac{k}{n}-$ отношение сигнал-шум; $k-$ количество информационных символов сообще-
ния. 
Произведение переходной вероятности на плотность распределения времени перехода из состояния $C_{2}$ в состояние $C_{3}$ определяется выражением

$$
P_{23} \varphi_{23}(t)=\left(1-P_{B}\right)^{L} \delta\left(t-\overline{T_{1}}\right),
$$

где $\bar{T}_{1}$ - среднее время передачи сообщения; $L$ - число элементов сообщения.

Переход $C_{3} \rightarrow C_{4}$ характеризуется временем полета снаряда и величиной отклонения снаряда от расчетной точки в плоскости цели. Будем полагать, что в силу случайностей, связанных с удалением РЭОб от средства поражения, время полета снаряда зададим экспоненциальным законом распределения, т.е.

$$
\varphi_{34}(t)=\frac{1}{\bar{T}_{2}} \exp \left(-\frac{t}{\bar{T}_{2}}\right),
$$

где $\bar{T}_{2}$ - время полета снаряда, полученное на основе усреднения по всем ситуациям, складывающихся значений при поражении РЭОб.

Время ожидания при переходе $C_{4} \rightarrow C_{5}$ и $C_{4} \rightarrow C_{6}$ равно 0.

Вероятности переходов $C_{7} \rightarrow C_{3}$ и $C_{9} \rightarrow C_{3}$ равны 1.

Переходы $C_{5} \rightarrow C_{7}, C_{5} \rightarrow C_{8}, C_{6} \rightarrow C_{9}, C_{6} \rightarrow C_{10}$ по сути представляют из себя процесс доразведки.

С учетом полученных переходных вероятностей и плотностей распределения времени переходов конечное выражение для вероятности поражения объекта принимает вид

$$
\begin{aligned}
& P_{10}(t)=L_{s}^{-1}\left\{\frac{1}{s} \cdot \frac{P_{0 i} \cdot P_{0 D} \cdot \exp \left(-s\left[\bar{T}_{\hat{i} a \dot{ }}+\bar{T}_{0}+\bar{T}_{1}\right]\right) \cdot\left[1-2 F\left(\frac{h_{x}}{\sigma_{x}}\right)\right]\left[1-2 F\left(\frac{h_{y}}{\sigma_{y}}\right)\right]}{\left(1-\left(1-P_{0 D}\right) \cdot \exp \left(-s \bar{T}_{i a i}\right)\right) \cdot\left(1-\left(1-\left[1-2 F\left(\frac{h_{x}}{\sigma_{x}}\right)\right]\left[1-2 F\left(\frac{h_{y}}{\sigma_{y}}\right)\right]\right) \cdot \exp \left(-s \bar{T}_{0}\right)\right)} \times\right. \\
& \left.\times \frac{P_{i i \hat{\delta}}^{i} \cdot\left(1-P_{B}\right)^{L} \cdot \frac{1}{\bar{T}_{2}} /\left(s+\frac{1}{\bar{T}_{2}}\right) \cdot D^{i i \hat{\delta} \delta}}{\left(1-\left(1-\left(1-P_{B}\right)^{L}\right) \cdot e^{-s T_{1}}\right) \cdot\left(1-\frac{1}{\bar{T}_{2}} /\left(s+\frac{1}{\bar{T}_{2}}\right) \cdot\left(\left(1-F_{i \hat{i} \delta}\right) \cdot\left(1-P_{i i \hat{\delta}}^{i}\right)+P_{i i \hat{\delta}}^{i} \cdot\left(1-D^{i \hat{\imath} \delta}\right)\right)\right)}\right\} .
\end{aligned}
$$

На графиках представлены зависимости вероятности поражения, полученные для следующих исходных данных: $D=0,9 ; F=10^{-4} ; \bar{T}_{a}=40$ мкс; $\sigma_{x, y}=20 \mathrm{м} ; h_{x, y}=40 \mathrm{м}$ - требуемые размеры области неопределенности для подсистемы поражения; для выбранного кода Голея $n=24$, $k=12, t=3 ; q=5 ; \bar{T}_{1}=1 \mathrm{мc} ; P_{n o p}^{n}=0,8-$ полигонная вероятность поражения; $D^{\text {nоp }}=0,9-$ условная вероятность правильного решения при поражении; $F^{\text {пор }}=10^{-3}-$ условная вероятность ложной тревоги.

Анализ зависимостей, представленных на рис. 5, позволяет сделать следующие выводы.

Вероятностно-временные показатели уничтожения излучающего объекта существенным образом зависят от динамических и вероятностных характеристик средств огневого поражения. Так, уменьшение полигонных значений вероятности поражения с 0,8 до 0,6 приводит к увеличению времени, необходимого для достижения заданного уровня конечных показателей более чем в 2 раза.

$$
-347-
$$




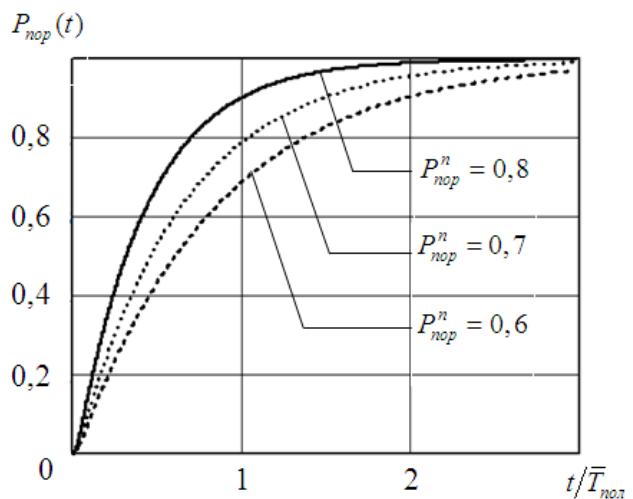

a)

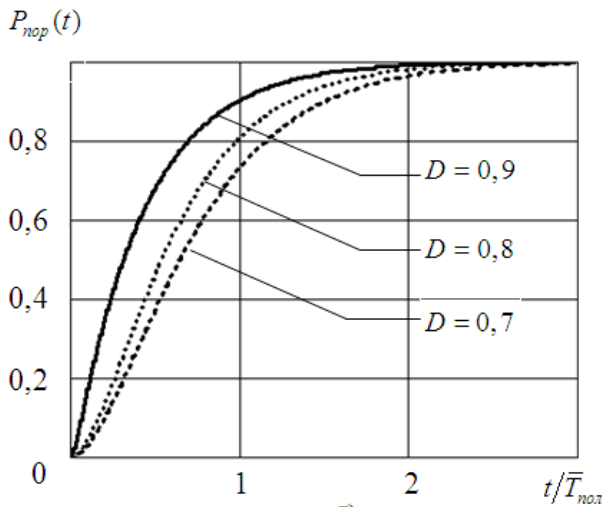

б)

Рис. 5. Графики зависимостей вероятности поражения от относительного времени при различных вероятностных значениях подсистем: а - подсистемы огневого поражения; б - подсистемы обнаружения

Динамические характеристики процессов поиска и координатометрии, а также их «точечные» вероятностные показатели (условные вероятности правильного обнаружения и ложной тревоги, функция распределения оценочных значений координат излучаемого РЭС) в случае сопоставимого значения среднего времени их реализации с динамическими параметрами полета средства поражения в значительной мере влияют на величину конечного показателя либо на временные параметры поражения. Так, при уменьшении условной вероятности правильного обнаружения с 0,9 до 0,7 вероятность поражения цели уменьшается на 17 \% (при неизменном времени вероятностных отсчетов).

Таким образом, рассмотренный подход позволяет с единых методических позиций за счет использования математического аппарата полумарковских случайных процессов рассмотреть и взаимно увязать по вероятностным показателям процессы поиска, координатометрии, передачи информации на средства огневого поражения и уничтожения излучающего РЭОб. Результаты исследования возможно использовать как для оценки эффективности каждой составляющей моделируемого процесса, так и для формирования требований к соответствующим подсистемам по значениям показателя уничтожения РЭОб.

\section{Список литературы}

[1] Модели информационного конфликта средств поиска и обнаружения / отв. ред. Ю.Л. Козирацкий. Москва: Радиотехника, 2013. 231 с. [Models of conflict information search and discovery tools / Editor J.L. Koziratsky. Moscow: Radiotechnics, 2013. 231 (in Russian)]

[2] Модели пространственного и частотного поиска / отв. ред. Ю.Л. Козирацкий. Москва: Радиотехника, 2014. 342 c. [Models of spatial and frequency search / Editor J.L. Koziratsky. Moscow: Radiotechnics, 2014. 342 (in Russian)]

[3] Паринов М.Л., Балаин С.Е., Петренков С.В. Способ координатометрии наземных источников радиоизлучения с режекцией входного потока по пространственному признаку. Вестник BBA. Bbin. 2(23). 2015. C. 390. [Parinov M.L., Balain S.E., Petrenko S.V. Method koordinatometrii land-based sources of radio emission from the input stream notch on spatial basis. 
Journal of Military Training and Research Center of the Air Force «Air Force Academy» №2(23), 2015, 390 (in Russian)]

[4] Козирацкий Ю.Л., Козирацкий А.Ю. и др. Способ пространственного мониторинга источников электромагнитного излучения. Патент № 2540126 от 25.09.2013. [Koziratsky Y.L., Koziratsky A.Y. et al. A method of monitoring a spatial electromagnetic radiation sources. Patent №2540126 of 09.25.2013. (in Russian)]

[5] Козирацкий Ю.Л., Паринов М.Л., Петренков С.В. Оценка влияния ошибок синхронизации опорных генераторов на точность определения направления на источник радиоизлучений с помощью забрасываемых пеленгационных элементов. Материалы XV Международной научно-методической конференции «Информатика: проблемы, методология, технологии». 1213 февраля 2015 г. Т. 3. Воронеж: ВГУ, 2015. С. 124-127. [Koziratsky Y.L., Parinov M.L., Petrenko S.V. Assessing the impact of local oscillator synchronization errors on the accuracy of determining the direction of the source of radio emissions via pelted direction-finding elements. Proceedings of the XV International scientific-methodical conference "Computer science: problems, methodology, and technology." February 12-13, 2015. Vol. 3. Voronezh: Voronezh State University, 2015, 124-127 (in Russian)]

[6] Козирацкий Ю.Л., Паринов М.Л., Петренков С.В., Сербов Д.А. Оценка влияния точности позиционирования забрасываемых пеленгационных элементов на ошибки определения направления на источник радиоизлучений. Вестник BBA. Bыn. 2(23), 2015. С. 319. [ Koziratsky Y.L, Parinov M.L., Petrenko S.V., Serbov D.A. Assessing the impact of the positioning accuracy of the direction-finding elements pelted error determining the direction of the source of radio emissions. Journal of Military Training and Research Center of the Air Force «Air Force Academy» № 2(23), 2015, 319 (in Russian)] 